\title{
miR-335-5p Regulates Cell Cycle and Metastasis in Lung Adenocarcinoma by Targeting CCNB2
}

This article was published in the following Dove Press journal: OncoTargets and Therapy

\section{Xiyong Wang \\ Huaiqing Xiao \\ Dongqiang Wu \\ Dongliang Zhang \\ Zhihao Zhang (ID}

Department of Thoracic Surgery, China Coast Guard Hospital of the People's Armed Police Force, Jiaxing 3/4000,

People's Republic of China
Correspondence: Zhihao Zhang Department of Thoracic Surgery, China Coast Guard Hospital of the People's Armed Police Force, No. 16 Nanhu Road, Jiaxing 3/4000, People's Republic of China Tel +86-13505736019

Email zzhhaomail234@I63.com
Background: Lots of studies have shown that cyclin disorders can promote tumor development. This study aims to investigate the biological function and molecular mechanism of CCNB2 in lung adenocarcinoma (LUAD).

Methods: LUAD data were downloaded from GEO database and TCGA-LUAD database. Differential analysis was conducted to find the differentially expressed miRNAs and mRNAs, while targeted prediction was done for the access of potential target mRNAs. Gene expression level was detected by qRT-PCR and Western blot in human LUAD cell lines A-427, A549, Calu-3, PC-9 and human bronchial epithelial cell line BEAS-2B. MTT, colony formation, Transwell and flow cytometry assays were used to detect cell proliferation, metastasis, and cell cycle changes of PC-9 cell line. The dual-luciferase reporter gene was used to detect the targeted binding relationship of the target miRNA and mRNA.

Results: CCNB2 was highly expressed and served as a biomarker indicating poor prognosis in LUAD patients. Cell function experiments confirmed the inhibitory effects of silencing CCNB2 on the proliferation, migration and invasion of LUAD cells and cell cycle was blocked in the G0/G1 phase. In addition, with regard to the regulatory mechanism, we demonstrated that miR-335-5p had binding sites with 3'-UTR of CCNB2, indicating that miR-335-5p could target the regulation expression of CCNB2. In subsequent cell function tests, overexpression of miR-335-5p inhibited the proliferation and metastasis of cancer cells, and the rescue experiments also verified that miR-335-5p could reverse the promotion of CCNB2 overexpression on the progress of cancer cells.

Conclusion: In summary, our results revealed that miR-335-5p could target the downregulation of CCNB2 to inhibit the occurrence and development of LUAD. Keywords: LUAD, CCNB2, miR-335-5p, proliferation, metastasis

\section{Introduction}

Lung adenocarcinoma (LUAD) mainly arises from small airway epithelial and type II alveolar cells ${ }^{1}$ and is one of the main subtypes of non-small cell lung cancer (NSCLC), accounting for $40 \%$ of lung cancer. ${ }^{2,3}$ According to the statistics of the national tumor registration points, LUAD accounted for $43.36 \%$ and squamous cell carcinoma accounted for $32.23 \%$ of the NSCLC patients in China from 2002 to 2012. ${ }^{4}$ It is obvious that LUAD has become the most common pathological type of NSCLC. In addition, despite significant advances in early detection and treatment, the prognosis of LUAD patients remains poor, with a 5-year survival rate less than $20 \%{ }^{5,6}$ Therefore, it is warranted to discover and develop new strategies for accurate diagnosis and effective treatment of LUAD. 
Cyclin family members have long been considered as key regulators of cell cycle progression, and abnormal expression of cyclin is closely related to tumor development and progression. ${ }^{7,8}$ For example, CCNA2 plays a dual regulatory role in modulating CDK6 and METmediated cell-cycle pathway and EMT progress in bladder cancer. ${ }^{9}$ CCNE1 is a potential target for ovarian cancer treatment. ${ }^{10}$ And HOXC13 promotes proliferation of LUAD via modulation of CCND1 and CCNE1. ${ }^{11}$ These studies have indicated that cyclins play an important role in regulating cancer progression accompanied by cell cycle changes.

CCNB2 as a member of cyclin family, plays a key role in $\mathrm{G} 2 / \mathrm{M}$ transformation and has been found to be upregulated in human cancers. ${ }^{12,13}$ For instance, CCNB2 overexpression in human liver cancer is associated with poor $\operatorname{prognosis}^{14}$ and the decrease of CCNB2 expression inhibits metastasis and invasion of bladder cancer. ${ }^{7}$ In addition, by analyzing Gene Expression Omnibus (GEO) database and constructing PPI network, Piao et al found that 14 genes including $\mathrm{CCNB} 2$ and $\mathrm{CDC} 20$ were associated with the prognosis of NSCLC patients. ${ }^{15}$ Based on bioinformatics, $\mathrm{Ni}$ et al obtained 15 genes including CCNB2 and EGR1 that were expected to be potential biomarkers and candidate targets for LUAD, which would be helpful to the diagnosis and treatment of LUAD. ${ }^{16}$ However, the specific mechanism of CCNB2 regulating the development of LUAD remains elusive.

In this study, we found that CCNB2 may play a critical role in the progression of LUAD and is likely to be regulated by miR-335-5p. In addition, the increase of CCNB2 expression is associated with the downregulation of related tumor suppressor factors in LUAD cells, while silencing CCNB2 inhibits the growth of LUAD cells. Therefore, exploring the molecular mechanism of CCNB2 in LUAD will help us to further understand the process of LUAD.

\section{Materials and Methods}

\section{Bioinformatics}

GSE75037 (Normal: 83, Tumor: 83) and GSE130779 (Normal: 8, Tumor: 8) datasets were downloaded from the GEO database (https:/www.ncbi.nlm.nih.gov/geo/). The batch correction of the datasets was performed by "sva" package, and the differential analysis between normal group and tumor group was conducted by "edgeR" package $(|\log \mathrm{FC}|>2.5, \quad$ padj $<0.05)$ to obtain the differentially expressed mRNAs (DEmRNAs). Meanwhile, the profiles of mature miRNA (Normal: 46, Tumor: 521) and mRNA (Normal: 59, Tumor: 535) along with clinical data were downloaded from TCGA-LUAD (https://portal.gdc.cancer. gov/). The differential analysis on normal group and tumor group was analyzed by "edgeR" package $(|\log F C|>1$, padj $<0.05)$ to obtain the differentially expressed miRNAs (DEmiRNAs). The upstream miRNAs of target mRNA were predicted using mirDIP (http://ophid.utoronto.ca/mirDIP/ index.jsp\#r) and starBase (http://starbase.sysu.edu.cn/) databases, and the miRNAs with binding sites of target mRNA were obtained by intersecting with DEmiRNAs. The "survival" package was used for survival analysis of target mRNA and corresponding miRNAs in TCGA-LUAD dataset, and for clinical staging analysis of target mRNA. GSEA was used for pathway enrichment analysis of target mRNA to study the mechanism of target miRNA and its target genes affecting LUAD.

\section{Cell Lines and Culture}

Human LUAD cell lines A-427 (BNCC341432), A549 (BNCC100215), Calu-3 (BNCC338514), PC-9 (BNCC340767) and human bronchial epithelial cell line BEAS -2B (BNCC338205) were all purchased from BeNa Culture Collection (BNCC, Beijing, China). All cell lines were cultured in Dulbecco's Modified Eagle Medium (DMEM; sigma, USA) containing $10 \%$ fetal bovine serum (FBS; Hyclone; GE Healthcare Life Sciences, Logan, UT, USA), $100 \mu / \mathrm{mL}$ streptomycin (Gibco; Thermo Fisher Scientific, Inc.) and $100 \mu / \mathrm{mL}$ penicillin (Gibco; Thermo Fisher Scientific, Inc.), then nurtured in $5 \% \mathrm{CO}_{2}$ at $37{ }^{\circ} \mathrm{C}$. The cells used in this study were all at 2-4 passages after recovery.

\section{Cell Transfection}

Mimic NC, miR-335-5p mimic, sh-NC and sh-CCNB2 (sh-CCNB2-1, sh-CCNB2-2, sh-CCNB2-3), oe-NC and oe-CCNB2 were obtained from GenePharma (Shanghai, China) and transfected into LUAD cell lines by Lipofectamine 2000 (Thermo Fisher Scientific, Inc.) according to the manufacturer's instructions. All cells were cultured in complete medium for at least 24 $\mathrm{h}$ before transfection, and rinsed with phosphate buffered saline (PBS, $\mathrm{pH}$ 7.4) before transient transfection.

\section{RNA Extraction and qRT-PCR}

Total RNA was isolated from cells using Trizol reagent (Invitrogen) according to the instructions. Reverse 
transcription (RT) and qRT-PCR were performed using the PrimeScript RT reagent kit (Takara, Dalian, China) and SYBR Prime Script RT PCR kit (Takara, Dalian, China), respectively. miR-335-5p and CCNB2 used U6 and GAPDH as internal references, respectively. The results were calculated using the $2^{-\Delta \Delta \mathrm{Ct}}$ method. The primer sequences were listed in Table 1.

\section{Western Blot (WB)}

The total proteins were loaded into sodium dodecyl sulfate polyacrylamide gel electrophoresis (SDS-PAGE) and transferred to the nitrocellulose membranes (Amersham, USA) blocked with 5\% skim milk powder at room temperature for $1 \mathrm{~h}$. Then the membranes were incubated with CCNB2 rabbit polyclonal antibodies (ab185622, 1:1000, abcam, Cambridge, UK) and GAPDH rabbit polyclonal antibodies (ab9485, 1:2500, abcam, Cambridge, UK) at $4{ }^{\circ} \mathrm{C}$ overnight and washed with PBST buffer (PBS buffer containing 0.1\% Tween-20) for $10 \mathrm{~min}$ for three times. Horseradish peroxidase-labeled secondary antibody goat anti-rabbit IgG (ab6721, 1:2000, abcam, Cambridge, UK) was added to the membranes for incubation at room temperature for $1 \mathrm{~h}$. The membranes were washed with PBST buffer for $10 \mathrm{~min}$ for 3 times. Optical luminometer (GE, USA) was utilized to detect immunoactivity.

\section{MTT Assay}

After transfection for $48 \mathrm{~h}$, the cells were digested with trypsin and then inoculated in 96 -well plates $\left(5 \times 10^{3}\right.$ cells/ well). At $24 \mathrm{~h}, 48 \mathrm{~h}$ and $72 \mathrm{~h}, 10 \mu \mathrm{L}$ MTT reagent with concentration of $5 \mathrm{mg} / \mathrm{mL}$ was added to cells for incubation at $37^{\circ} \mathrm{C}$ for $4 \mathrm{~h}$. After the supernatant was discarded, cells were exposed to $200 \mu \mathrm{L}$ dimethyl sulfoxide (DMSO). The absorbance at $490 \mathrm{~nm}$ was measured using a microplate reader (Thermo Fisher Scientific).

Table I Primer Sequences

\begin{tabular}{|l|l|}
\hline Target Gene & Primer (5' -3') $^{\prime}$ \\
\hline miR-335-5p & $\begin{array}{l}\text { F:TGTTTTGAGCGGGGGTCAAGAGC } \\
\text { R:CTCTCATTTGCTATATTCA }\end{array}$ \\
\hline U6 & $\begin{array}{l}\text { F:CTCGCTTCGGCAGCACA } \\
\text { R:AACGCTTCACGAATTTGCGT }\end{array}$ \\
\hline CCNB2 & $\begin{array}{l}\text { F:CCGACGGTGTCCAGTGATTT } \\
\text { R:TGTTGTTTTGGTGGGTTGAACT }\end{array}$ \\
\hline GAPDH & $\begin{array}{l}\text { F:GGAGCGAGATCCCTCCAAAAT } \\
\text { R:GGCTGTTGTCATACTTCTCATGG }\end{array}$ \\
\hline
\end{tabular}

\section{Colony Formation Assay}

Cell proliferation was analyzed by colony formation assay. After $48 \mathrm{~h}$ of transfection, $1 \times 10^{3}$ cells were seeded on the plates to incubate at $37{ }^{\circ} \mathrm{C}$ for about 2 weeks. The cell colonies were fixed with $70 \%$ alcohol for $5 \mathrm{~min}$, and then stained with $0.5 \%$ crystal violet. The number of colonies was calculated.

\section{Flow Cytometry (FCM)}

The cell cycle was analyzed by FCM. Cells for the experiment were collected, treated with trypsin, washed with PBS, and then fixed with cold ethanol. Then propidium iodide (PI; Sigma, St Louis, MO, USA) was used to stain cells for $15 \mathrm{~min}$, and cell proportions of each period were analyzed by FCM (Beckman Coulter, Brea, CA).

\section{Transwell}

Cell migration and invasion tests were performed in Transwell chambers (Corning Inc., Corning, NY, USA) with a polycarbonate membrane. In Transwell migration assay, $1 \times 10^{5}$ cells were seeded in serum-free DMEM in the upper chambers, and the lower chambers contained $10 \%$ FBS. After incubation for about $10 \mathrm{~h}$, cells in the upper chambers were wiped off and cells in the lower chambers were stained with crystal violet at $25{ }^{\circ} \mathrm{C}$ for 1 min, observed and counted under a light microscope (Nikon, 100×). Cells from 5 randomly selected fields were counted and averaged. The procedures of Transwell invasion assay were the same as above, except that the upper chambers were coated with $20 \mu \mathrm{g}$ extracellular Matrix gel (Sigma-Aldrich; Merck KGaA).

\section{Dual-Luciferase Reporter Gene Assay}

The luciferase reporter vectors based on psicheck 2 were constructed including wild-type CCNB2 (CCNB2-WT) and mutant-type CCNB2 (CCNB2-MUT) at binding sites of miR-335-5p. HEK293T cells were seeded into 24-well plates and co-transfected with CCNB2-WT /CCNB2-MUT vector, miR-335-5p mimic/mimic NC via Lipofectamine 2000. After transfection for $48 \mathrm{~h}$, cell lysates were collected and the luciferase activity was measured by using dual-luciferase reporter gene assay system (Promega).

\section{Statistical Analysis}

GraphPad Prism 6.0 (Graphpad Software Inc.) was employed for statistical analysis. All data were presented 
as mean \pm standard deviation (SD). The difference between two groups was analyzed by $t$-test. Pearson $\chi^{2}$ test was used to analyze the relationship between expression of miR-335-5p and CCNB2. All the experiments in the study were repeated three times. $P<0.05$ or $P<0.01$ were considered statistically significant.

\section{Results}

\section{CCNB2 Is Highly Expressed in LUAD and Is Associated with Poor Prognosis}

The CCNB2 expression in LUAD cells was significantly higher than that in BEAS-2B cell line (Figure 1A and B). In addition, through the analysis of DEmRNA expression in GEO database, we found that CCNB2 was considerably highly expressed in tumor tissues (Figure 1C). The survival analysis of the TCGALUAD dataset showed that the survival time of patients with higher CCNB2 expression was remarkably lower (Figure 1D). Combined with the clinical information of patients, it was revealed that CCNB2 showed significant differences at different clinical stages (Figure 1E), $\mathrm{N}$ stages (Figure $1 \mathrm{~F}$ ), and $\mathrm{T}$ stages (Figure $1 \mathrm{G}$ ) of LUAD, and the expression level was elevated with the increase of tumor stage. Therefore, we believed that CCNB2 might act as an oncogene in LUAD and was associated with poor prognosis.

\section{Down-Regulation of CCNB2 Inhibits the Proliferation, Migration, Invasion and Cell Cycle of LUAD Cells}

The mRNA and protein expressions of CCNB2 were significantly decreased in PC-9 cells transfected with 3 sets of sh-CCNB2 (Figure 2A and B). We selected sh-CCNB2-3 with the most significant decline for follow-up experiments. The results of MTT (Figure 2C), colony formation (Figure 2D) and Transwell (Figure 2E and F) assays observed that reduced CCNB2 expression resulted in the inhibition of the proliferation activity, colony forming, migration and invasion abilities of PC-9 cells.

In addition, single-gene enrichment analysis showed that CCNB2 was considerably enriched in the cell cycle pathway (Figure $2 \mathrm{G}$ ). Thus, we measured the cell cycle by FCM. The results exhibited that compared with NC group, the proportion of PC-9 cells in G0/G1 phase was significantly higher, while that in $\mathrm{S}$ and $\mathrm{G} 2 / \mathrm{M}$ phases was significantly lower in sh-CCNB2 group (Figure 2H), indicating that the decreased CCNB2 expression inhibited the proliferation of cancer cells.

\section{CCNB2 Is a Target Gene of miR-335-5p}

To gain more insight into the mechanism of CCNB2 in LUAD, we conducted in-depth mining and analysis of its upstream regulatory factors. Firstly, according to the data in
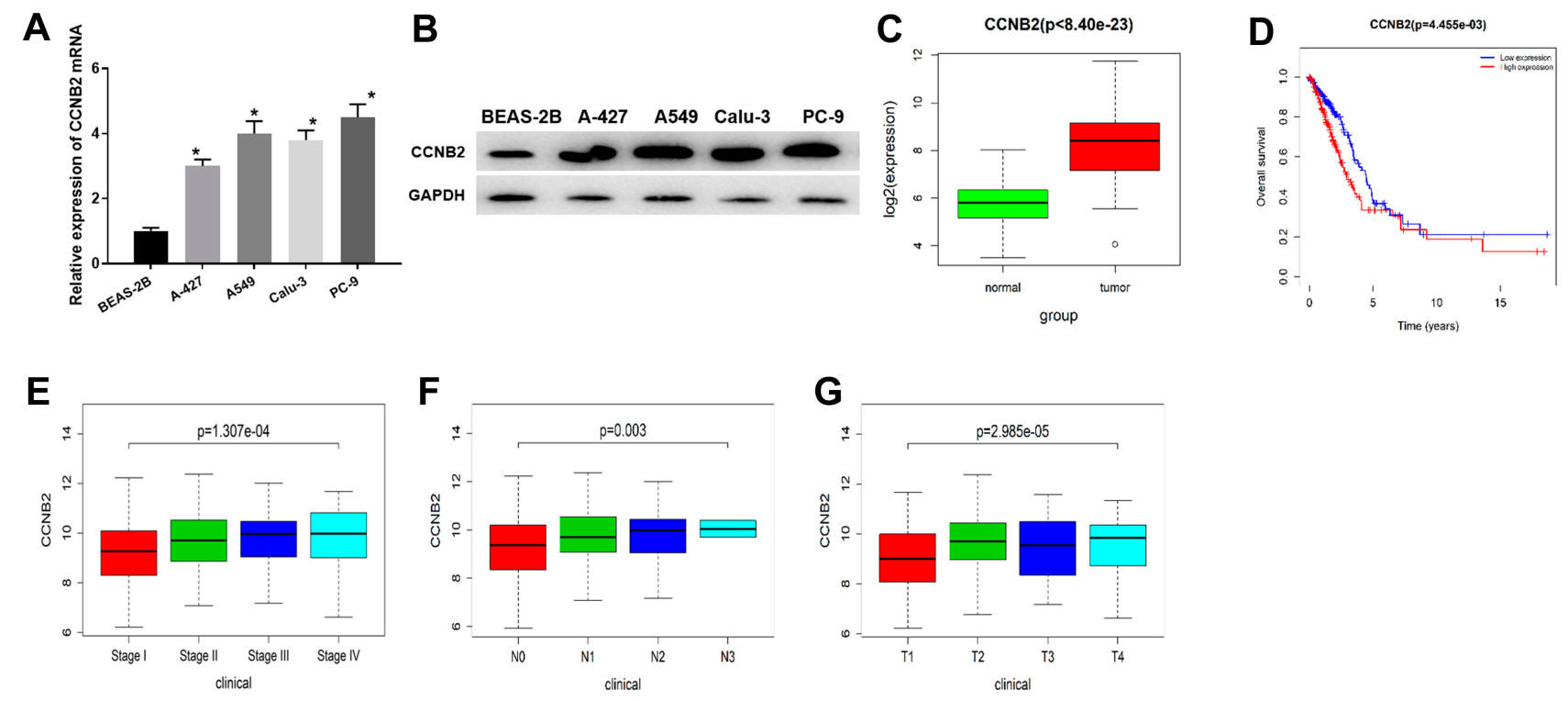

Figure I The expression and prognosis of CCNB2 in LUAD. (A and B) mRNA and protein expressions of CCNB2 in BEAS-2B and LUAD cell lines (A-427, A549, Calu-3, PC-9); (C) The expression of CCNB2 was low in normal samples (green) and high in tumor samples (red); (D) Survival curves of CCNB2 expression for prognosis in the TCGA-LUAD dataset. Red indicates high expression group and blue indicates low expression group; (E-G) Box plots of CCNB2 expression in different clinical stages, N stages and T stages of LUAD. Note: *Indicates $P<0.05$. 


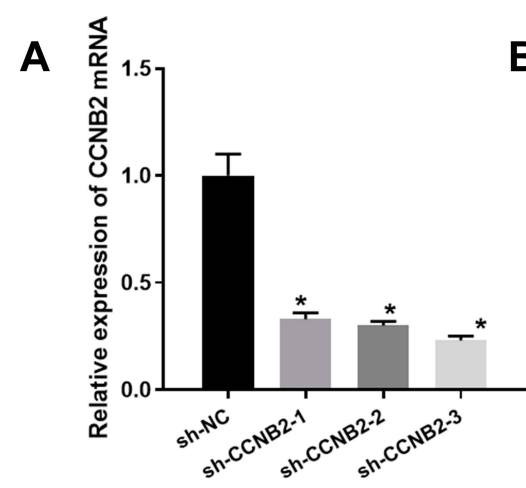

B

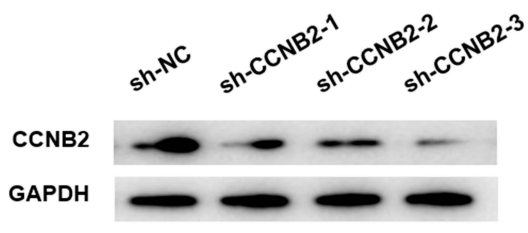

C

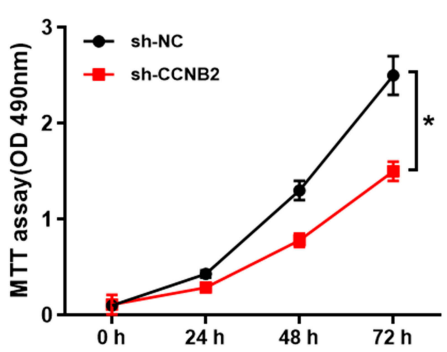

D

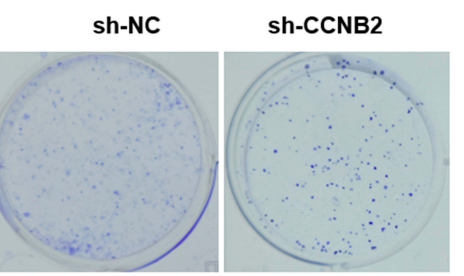

E
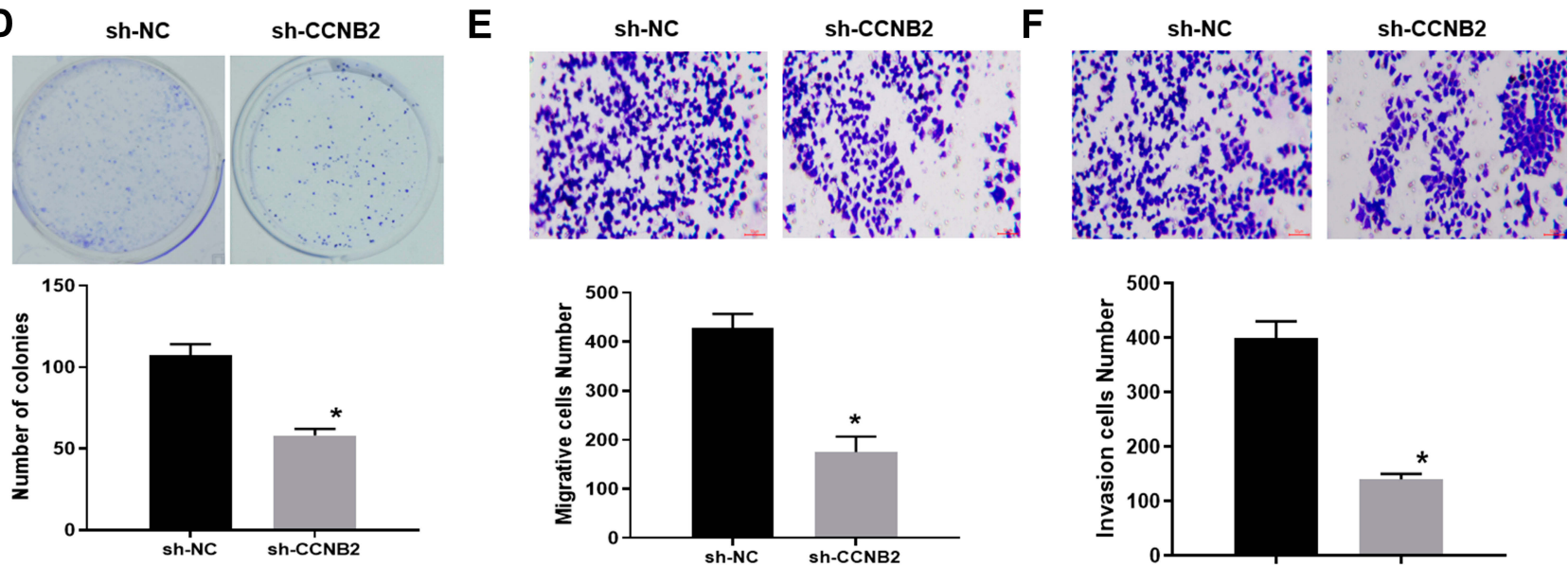

$\mathbf{G}$

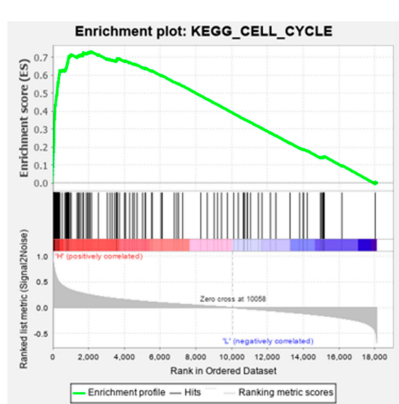

H
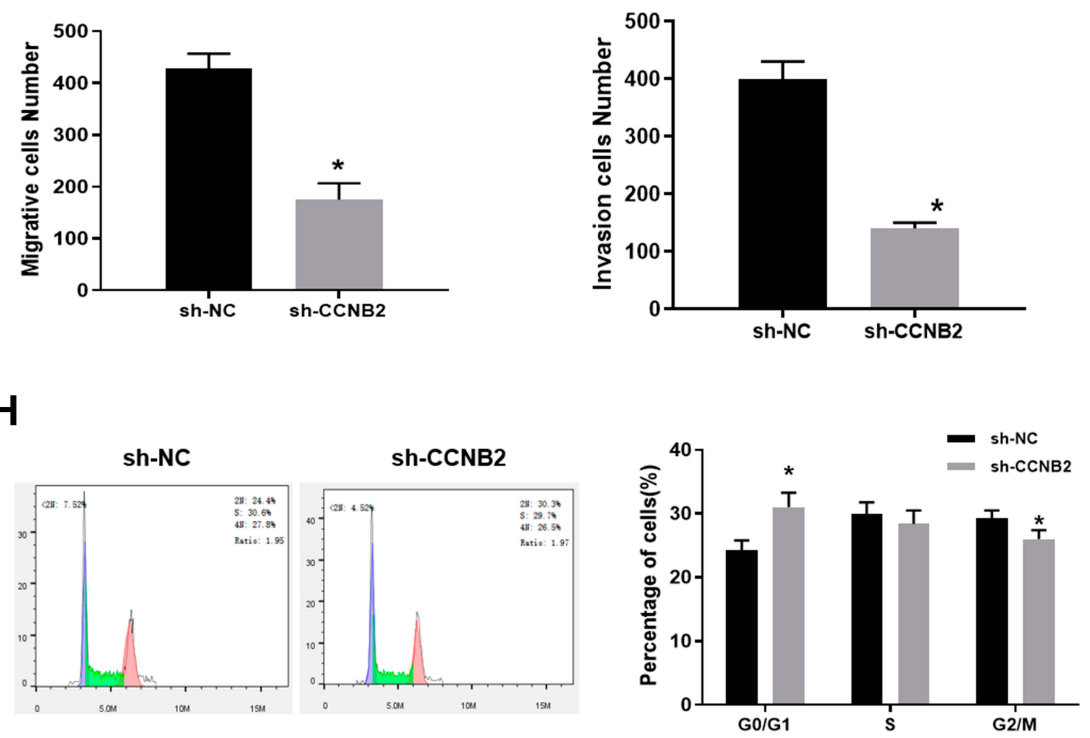

Figure 2 Low expression of CCNB2 inhibits the proliferation, metastasis and cell cycle of LUAD cells. (A and B) mRNA and protein levels in CCNB2-silenced LUAD cells; (C) The MTT assay was used to measure the cell viability of LUAD cells at $24 \mathrm{~h}, 48 \mathrm{~h}$ and $72 \mathrm{~h}$, respectively; (D) The proliferation of LUAD cells was determined by colony formation assay; (E and F) Transwell assay was used to determine the migration and invasion abilities of LUAD cells; (G) GSEA pathway enrichment analysis results of CCNB2; (H) FCM was used to analyze the proportion of cells in each cell cycle phase of LUAD cells (CCNB2-silenced cells and NC cells).

Note: *Indicates $P<0.05$.

TCGA-LUAD, a total of 299 DEmiRNAs were obtained by "edgeR" for differential analysis (Figure 3A). Then we predicted the upstream miRNAs of CCNB2 through mirDIP and starBase databases, and the potential regulatory factor miR-335-5p of CCNB2 was obtained from the intersection of the down-regulated 79 DEmiRNAs (Figure 3B). Pearson correlation analysis displayed that $\mathrm{miR}-335-5 \mathrm{p}$ was negatively correlated with CCNB2 (Figure 3C) and extremely significantly low-expressed in tumor tissues (Figure 3D), which was consistent with the results in cell lines (Figure 3E). Moreover, TCGA-LUAD dataset survival analysis also indicted that patients with high expression of miR-335-5p survived significantly longer than those with low expression (Figure 3F).

miRDIP and starBase databases were applied and found that miR-335-5p and CCNB2 had targeted binding regions (Figure $3 \mathrm{G}$ ). In order to prove the targeted regulatory relationship between miR-335-5p and CCNE2, we firstly detected the mRNA and protein expressions of CCNB2 in miR-335-5p mimic group and NC group in 

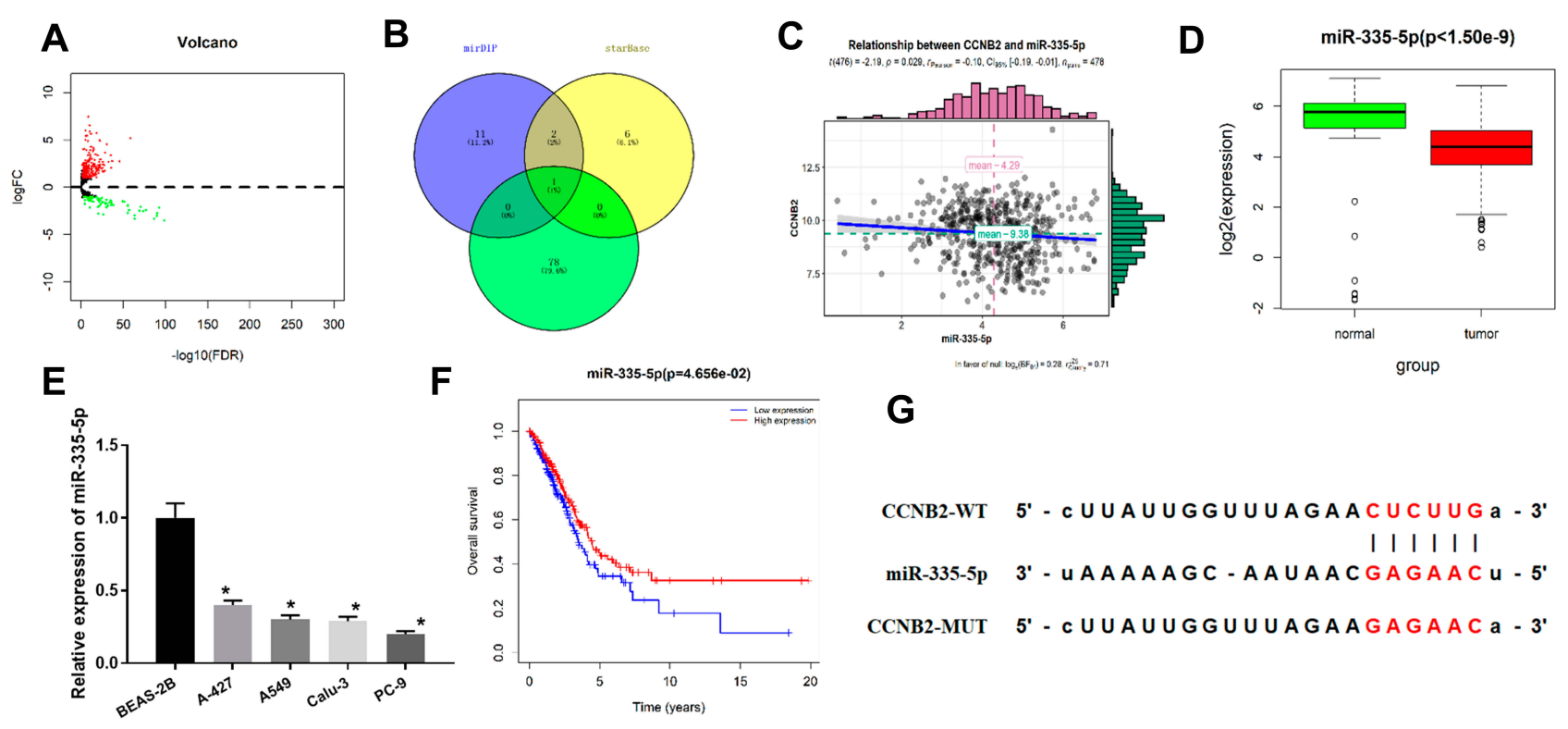

G
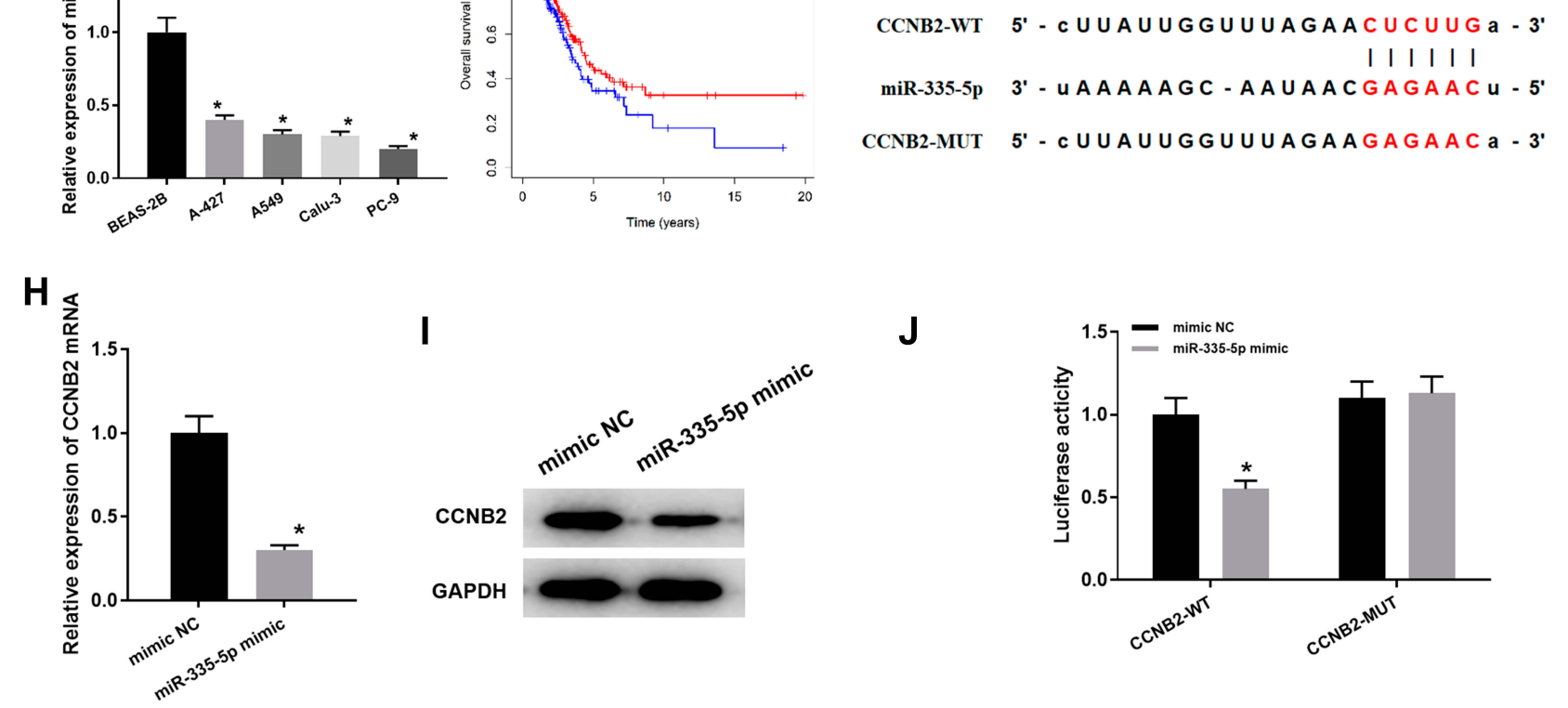

Figure 3 miR-335-5p targeted down-regulates CCNB2. (A) DEmiRNA volcano map of normal group and tumor group in TCGA-LUAD dataset; (B) Venn diagram of predicted upstream down-regulated DEmiRNAs for CCNB2; (C) Pearson correlation analysis of CCNB2 and miR-335-5p; (D) The miR-335-5p expression was highly expressed in normal samples (green) and lowly expressed in tumor samples (red); (E) Expression of miR-335-5p in BEAS-2B cell line and LUAD cell lines (A-427, A549, Calu-3, PC-9); (F) Survival curves of miR-335-5p expression for prognosis. Red indicates high expression group and blue indicates low expression group; (G) Binding sites of miR-335-5p and 3 'UTR of CCNB2; (H and I) qRT-PCR and WB were used to detect the effects of miR-335-5p expression on CCNB2 mRNA and protein levels; (J) Dualluciferase reporter gene assay was used to determine the targeted binding of miR-335-5p and CCNB2.

Note: *Indicates $P<0.05$.

PC-9 cells, and the results showed that the expression of CCNB2 was significantly down-regulated when miR-335$5 \mathrm{p}$ was overexpressed (Figure $3 \mathrm{H}$ and I). The result of Dual-luciferase reporter gene assay showed that miR-335$5 \mathrm{p}$ mimic significantly inhibited luciferase activity in CCNB2-WT group (Figure 3J). Our results demonstrated that CCNB2 was a target gene of miR-335-5p and was negatively regulated by miR-335-5p in LUAD.

\section{Overexpression of miR-335-5p Inhibits Proliferation, Migration and Invasion of LUAD Cells Which Can Be Reversed by CCNB2}

Based on the regulation of miR-335-5p on CCNB2, we further investigated the effects of miR-335-5p expression on cell functions. We firstly examined the overexpression efficiency of miR-335-5p and CCNB2 (Figure 4A). The results of MTT (Figure 4B), colony formation (Figure 4C) and Transwell (Figure 4D and E) assays exhibited that compared with the NC group, the overexpression of miR-335-5p significantly inhibited the cell activity, proliferation, migration and invasion, while with a concomitant of overexpressed CCNB2 would reverse the inhibitory effects of miR-335-5p overexpression in LUAD cells. In addition, the cell cycle detected by FCM also reflected that overexpression of miR-335-5p blocked cells in the G0/G1 phase to suppress cancer cell proliferation (Figure 4F). In summary, our experiments showed that miR-335-5p inhibited cell function, and its inhibitory effect could be reversed by oe-CCNB2 in LUAD. 


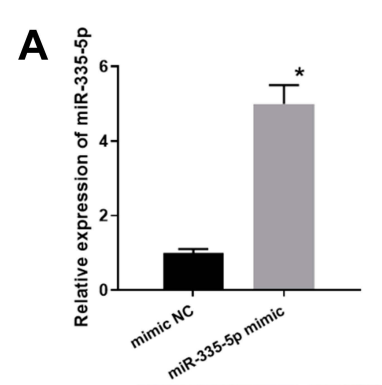

C
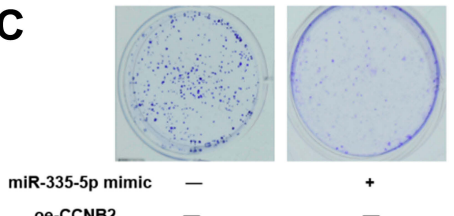

oe-CCNB2
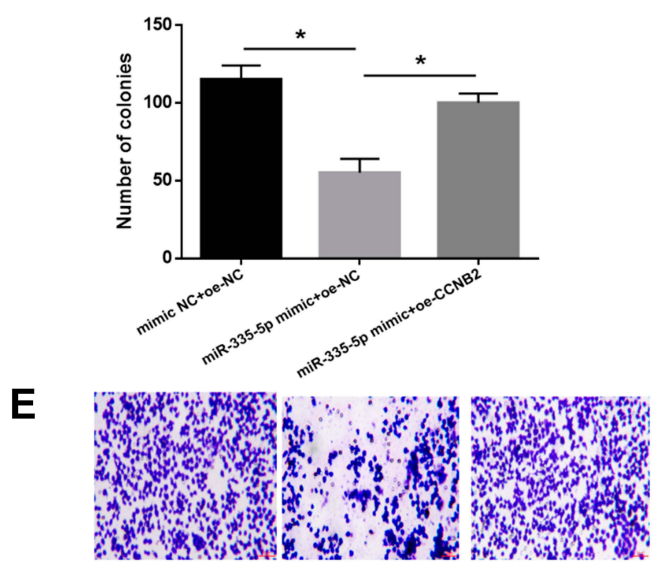

miR-335-5p mimic oe-CCNB2

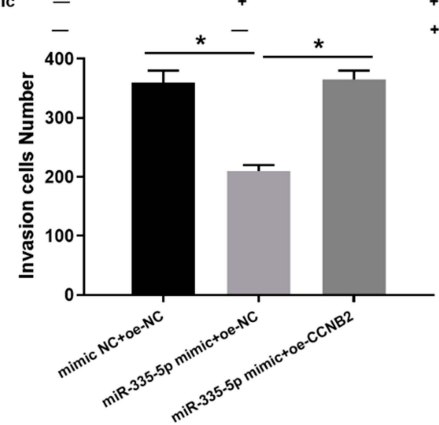

B

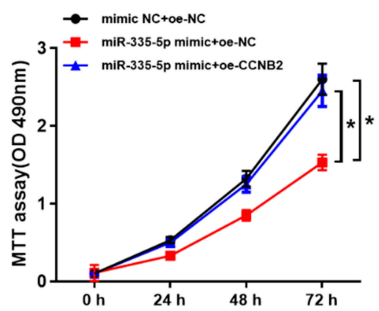

D

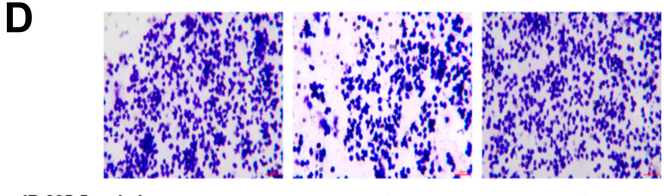

miR-335-5p mimic oe-CCNB2
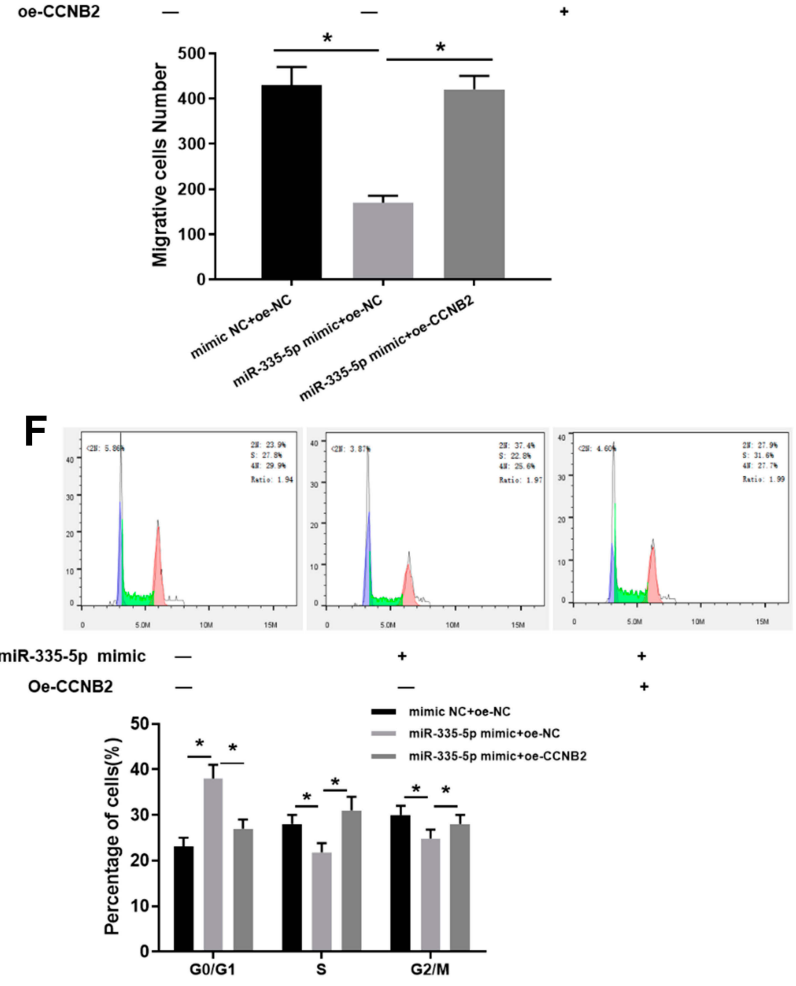

Figure 4 Overexpression of miR-335-5p inhibits LUAD development which can be reversed by CCNB2. (A) Expression of miR-335-5p and CCNB in transfected cells was detected by qRT-PCR; (B) MTT assay was used to determine the cell viability at $24 \mathrm{~h}, 48 \mathrm{~h}$, and $72 \mathrm{~h}$, respectively; (C) The proliferation of LUAD cells was determined by colony formation assay; ( $\mathbf{D}$ and $\mathbf{E}$ ) Transwell assay was used to determine the migration and invasion ability of LUAD cells; (F) The proportion of cells in each cell cycle stage of LUAD cells was analyzed by FCM.

Note: *Indicates $P<0.05$.

\section{Discussion}

In this study, we confirmed that CCNB2 was significantly upregulated in LUAD. Cytological studies showed that silencing CCNB2 inhibited the proliferation and metastasis of LUAD cells. Mechanism studies revealed that the expression of CCNB2 could be regulated by miR-335-5p.

CCNB2 has been reported to be up-regulated in many tumors, such as gastric cancer, ${ }^{17}$ hepatocellular carcinoma, ${ }^{18}$ and glioma. ${ }^{19}$ In this study, through the analysis of
GSE75037 and GSE130779 mRNA datasets of LUAD in GEO database, we discovered significant differences in CCNB2 expression in normal group and tumor group. Meanwhile, CCNB2 mRNA and protein levels also confirmed that CCNB2 was highly expressed in LUAD cell lines. Studies have indicated that CCNB2 may be a potential biomarker of unfavorable prognosis over shortterm follow-up in breast cancer. ${ }^{20}$ Therefore, we used the "survival" package to carry out survival analysis and clinical 
stage analysis on CCNB2 in TCGA-LUAD dataset, and observed that the survival prognosis of patients with high CCNB2 expression was obviously poor, and there were significant differences in different clinical stages, $\mathrm{N}$ stages and T stages. It was suggested that CCNB2 could be served as a prognostic factor for survival and early diagnosis of LUAD. Furthermore, we also verified the effects of CCNB2 on cell functions by silencing its expression in PC-9 cell line, and found that silencing CCNB2 remarkably inhibited the proliferation, migration and invasion abilities of cells and the cell proportion was significantly increased in $\mathrm{G} 0 / \mathrm{G} 1$ phase. Our data further confirmed that CCNB2 played an important role in the process of LUAD.

miRNA plays an important role in many biological processes such as migration, differentiation, apoptosis and transformation. ${ }^{21}$ It induces translational suppression or post-transcriptional degradation of mRNA mainly through binding to the 3'UTR of the target gene mRNA. ${ }^{22,23}$ In order to further explore the upstream regulatory mechanism of $\mathrm{CCNB} 2$, we used bioinformatics to analyze miRNAs in TCGA-LUAD to obtain miR-335-5p, which had targeted binding sites with CCNB2 and was significantly down-regulated in LUAD. A series of experiments results showed that miR-335-5p could target the down-

regulation of CCNB2 in LUAD cell lines, indicating that it was likely to regulate the process of LUAD as a tumor suppressor.

miR-335-5p has been related to certain types of cancer. $^{24-26}$ miR-335-5p inhibits cell proliferation, migration and invasion in colorectal cancer through downregulating LDHB. $^{27}$ miR-335-5p targeting ICAM-1 inhibits invasion and metastasis of thyroid cancer cells. ${ }^{28}$ And miR-335-5p inhibits TGF- $\beta 1$-induced EMT in NSCLC via ROCK $1 .{ }^{29}$ In this study, we also verified whether miR-335-5p could affect the function of LUAD cells by regulating CCNB2 at the cellular level. The results showed that overexpression of miR-335-5p significantly inhibited the proliferation and metastasis of LUAD cell lines, and its inhibitory effect could be reversed when CCNB2 was overexpressed, which were in parallel to the trend of miR-335-5p effects in other tumors. Besides, miR-335-5p has also been reported to restore cisplatin sensitivity in ovarian cancer cells via targeting BCL2L $2,{ }^{30}$ which indicates that miR-335-5p may play an important role in the process of tumor treatment. In the future, we can also use the miR-335-5p/CCNB2 axis as a target site for LUAD molecular therapy, so as to improve the therapeutic effects and prognosis of LUAD patients.

CCNB2, as a key protein in the cell cycle, has a significant impact on the proliferation of cancer cells. Our study explored the mechanism of up-regulated CCNB2 in regulating the malignant process of LUAD as a target gene of miR-335-5p. In the future, CCNB2 can not only be used as a marker for the diagnosis of LUAD, but also may be able to improve the therapeutic effect of LUAD patients. And this may be a new direction for our future research. In conclusion, this study provides a new theoretical basis for molecular targeted therapy of LUAD in the future.

\section{Data Sharing Statement}

The data used to support the findings of this study are included within the article. The data and materials in the current study are available from the corresponding author on reasonable request.

\section{Author Contributions}

All authors contributed to data analysis, drafting and revising the article, gave final approval of the version to be published, and agreed to be accountable for all aspects of the work.

\section{Funding}

Not applicable.

\section{Disclosure}

The authors declare no conflicts of interest.

\section{References}

1. Zappa C, Mousa SA. Non-small cell lung cancer: current treatment and future advances. Transl Lung Cancer Res. 2016;5(3):288-300. doi:10.21037/tlcr.2016.06.07

2. Travis WD, Brambilla E, Noguchi M, et al. International association for the study of lung cancer/American Thoracic Society/European Respiratory Society: international multidisciplinary classification of lung adenocarcinoma: executive summary. Proc Am Thorac Soc. 2011;8(5):381-385. doi:10.1513/pats.201107-042ST

3. Moran C. Importance of molecular features of non-small cell lung cancer for choice of treatment. Am J Pathol. 2011;178(5):1940-1948. doi:10.1016/j.ajpath.2010.12.057

4. Zou XN, Lin DM, Wan X, et al. Histological subtypes of lung cancer in Chinese males from 2000 to 2012. Biomed Environ Sci. 2014;27 (1):3-9. doi:10.3967/bes2014.010

5. Ferlay J, Soerjomataram I, Dikshit R, et al. Cancer incidence and mortality worldwide: sources, methods and major patterns in GLOBOCAN 2012. Int $J$ Cancer. 2015;136(5):E359-E386. doi:10. 1002/ijc. 29210 
6. Wood SL, Pernemalm M, Crosbie PA, et al. Molecular histology of lung cancer: from targets to treatments. Cancer Treat Rev. 2015;41 (4):361-375. doi:10.1016/j.ctrv.2015.02.008

7. Lei C-Y, Wang W, Zhu Y-T, et al. The decrease of cyclin B2 expression inhibits invasion and metastasis of bladder cancer. Urol Oncol. 2016;34(5):237.e1. doi:10.1016/j.urolonc.2015.11.011

8. Peng X, Pan K, Zhao W, et al. NPTX1 inhibits colon cancer cell proliferation through down-regulating cyclin A2 and CDK2 expression. Cell Biol Int. 2018;42(5):589-597. doi:10.1002/cbin.10 935

9. Li J, Ying $\mathrm{Y}$, Xie $\mathrm{H}$, et al. Dual regulatory role of CCNA2 in modulating CDK6 and MET-mediated cell-cycle pathway and EMT progression is blocked by miR-381-3p in bladder cancer. FASEB J. 2019;33(1):1374-1388. doi:10.1096/fj.201800667R

10. Kanska J, Zakhour M, Taylor-Harding B, et al. Cyclin E as a potential therapeutic target in high grade serous ovarian cancer. Gynecol Oncol. 2016;143(1):152-158. doi:10.1016/j.ygyno.2016.07. 111

11. Yao Y, Luo J, Sun Q, et al. HOXC13 promotes proliferation of lung adenocarcinoma via modulation of CCND1 and CCNE1. Am J Cancer Res. 2017;7(9):1820-1834.

12. Qian X, Song X, He Y, et al. CCNB2 overexpression is a poor prognostic biomarker in Chinese NSCLC patients. Biomed Pharmacother. 2015;74:222-227. doi:10.1016/j.biopha.2015.08.004

13. Mo M-L, Chen Z, Li J, et al. Use of serum circulating CCNB2 in cancer surveillance. Int J Biol Markers. 2010;25(4):236-242. doi:10.5301/JBM.2010.6088

14. Li R, Jiang X, Zhang Y, et al. Cyclin B2 overexpression in human hepatocellular carcinoma is associated with poor prognosis. Arch Med Res. 2019;50(1):10-17. doi:10.1016/j.arcmed.2019.03.003

15. Piao J, Sun J, Yang Y, et al. Target gene screening and evaluation of prognostic values in non-small cell lung cancers by bioinformatics analysis. Gene. 2018;647:306-311. doi:10.1016/j.gene.2018.01.003

16. Ni KW, Sun GZ. The identification of key biomarkers in patients with lung adenocarcinoma based on bioinformatics. Math Biosci Eng. 2019;16(6):7671-7687. doi:10.3934/mbe.2019384

17. Shi Q, Wang W, Jia Z, et al. ISL1, a novel regulator of CCNB1, CCNB2 and c-MYC genes, promotes gastric cancer cell proliferation and tumor growth. Oncotarget. 2016;7(24):36489-36500. doi:10.186 32/oncotarget.9269

18. Zhu H-R, Yu X-N, Zhang G-C, et al. Comprehensive analysis of long non coding RNA messenger RNA microRNA co expression network identifies cell cycle related lncRNA in hepatocellular carcinoma. Int J Mol Med. 2019;44(5):1844-1854. doi:10.3892/ijmm.2019.4323
19. Xi X, Chu Y, Liu N, et al. Joint bioinformatics analysis of underlying potential functions of hsa-let-7b-5p and core genes in human glioma. J Transl Med. 2019;17(1):129. doi:10.1186/s12967-019-1882-7

20. Shubbar E, Kovács A, Hajizadeh S, et al. Elevated cyclin B2 expression in invasive breast carcinoma is associated with unfavorable clinical outcome. BMC Cancer. 2013;13:1. doi:10.1186/1471-2407-13-1

21. Bayoumi AS, Sayed A, Broskova Z, et al. Crosstalk between long noncoding RNAs and microRNAs in health and disease. Int $\mathrm{J} \mathrm{Mol}$ Sci. 2016;17(3):356. doi:10.3390/ijms17030356

22. Raitoharju E, Seppälä I, Oksala N, et al. Blood microRNA profile associates with the levels of serum lipids and metabolites associated with glucose metabolism and insulin resistance and pinpoints pathways underlying metabolic syndrome: the cardiovascular risk in Young Finns Study. Mol Cell Endocrinol. 2014;391(1-2):41-49. doi:10.1016/j.mce.2014.04.013

23. Plaisance-Bonstaff K, Renne R. Viral miRNAs. Methods Mol Biol. 2011;721:43-66

24. Zhang P, Yang X, Wang L, et al. Overexpressing miR-335 inhibits DU145 cell proliferation by targeting early growth response 3 in prostate cancer. Int J Oncol. 2019;54(6):1981-1994. doi:10.3892/ ijo. 2019.4778

25. Almanza G, Rodvold JJ, Tsui B, et al. Extracellular vesicles produced in B cells deliver tumor suppressor miR-335 to breast cancer cells disrupting oncogenic programming in vitro and in vivo. Sci Rep. 2018;8(1):17581. doi:10.1038/s41598-018-35968-2

26. Wang K, Jin W, Song Y, et al. LncRNA RP11-436H11.5, functioning as a competitive endogenous RNA, upregulates BCL-W expression by sponging miR-335-5p and promotes proliferation and invasion in renal cell carcinoma. Mol Cancer. 2017;16(1):166. doi:10.1186/ s12943-017-0735-3

27. Zhang D, Yang N. MiR-335-5p inhibits cell proliferation, migration and invasion in colorectal cancer through downregulating LDHB. J BUON. 2019;24(3):1128-1136.

28. Luo L, Xia L, Zha B, et al. miR-335-5p targeting ICAM-1 inhibits invasion and metastasis of thyroid cancer cells. Biomed Pharmacother. 2018;106:983-990. doi:10.1016/j.biopha.2018.07.046

29. Du W, Tang H, Lei $Z$, et al. miR-335-5p inhibits TGF- $\beta 1$-induced epithelial-mesenchymal transition in non-small cell lung cancer via ROCK1. Respir Res. 2019;20(1):225. doi:10.1186/s12931-019-1184-x

30. Liu R, Guo H, Lu S. MiR-335-5p restores cisplatin sensitivity in ovarian cancer cells through targeting BCL2L2. Cancer Med. 2018;7 (9):4598-4609. doi:10.1002/cam4.1682
OncoTargets and Therapy

\section{Publish your work in this journal}

OncoTargets and Therapy is an international, peer-reviewed, open access journal focusing on the pathological basis of all cancers, potential targets for therapy and treatment protocols employed to improve the management of cancer patients. The journal also focuses on the impact of management programs and new therapeutic agents and protocols on patient perspectives such as quality of life, adherence and satisfaction. The manuscript management system is completely online and includes a very quick and fair peer-review system, which is all easy to use. Visit http://www.dovepress.com/ testimonials.php to read real quotes from published authors. 